Published in final edited form as:

Semin Liver Dis. 2015 November ; 35(4): 355-360. doi:10.1055/s-0035-1567833.

\title{
Genome-Wide Association Studies and Liver Disease
}

\author{
Elizabeth K. Speliotes, MD, PhD, MPH ${ }^{1}$ \\ ${ }^{1}$ Divisions of Gastroenterology, and Computational Medicine and Bioinformatics, Department of \\ Internal Medicine, University of Michigan, Ann Arbor, Michigan
}

\begin{abstract}
Sequencing of the human genome has opened up many opportunities to learn about our own genetic susceptibilities to disease. In this Foreword to this issue of Seminars in Liver Disease, I provide some required background to understanding genome-wide association analyses in general, including a list of terms (Table 1) often used in such studies. Five areas of particular significance are then reviewed in detail in the articles that follow.
\end{abstract}

\section{Genetics beyond Mendel}

Many human traits including the risk of developing disease, progressing to more severe disease, and responding to treatments for disease are genetically influenced, but complex. Mendelian genetic conditions are typified by the presence of a genetic variant that invariably leads to a clearly noticeable phenotype or trait in individuals who carry it, often irrespective of other genetic variants or environmental influences. Indeed, most diseases (> 99\%) encountered in clinical practice are genetically influenced, but complex. For complex genetic traits, genetic variants increase the likelihood of developing a phenotype in combination with other genetic variants and environmental influences. The proportion of the trait variance explained by genetic factors is called its heritability and can be measured by various methods. Although not all traits have been formally characterized to determine their heritability, most do have a genetic component. Recent advances in our understanding of the human genome and in genotyping technologies now enable the identification of genetic variants that affect many human traits.

\section{Human Genetic Variation}

Sequencing of the human genome has revealed much similarity in the genetic code between individuals: More than $99.9 \%$ of the DNA between two individuals is identical. ${ }^{1-4}$ The remaining $0.1 \%$ constitutes variations in the genetic code at certain sites and is largely in the form of single nucleotide polymorphisms (SNPs); $90 \%$ of these are common (frequency > $1 \%$ ) in the population and $10 \%$ are rare (frequency $<1 \%$ ). 5,6 Once cataloged, these SNPs can be genotyped, which, unlike sequencing, does not characterize every nucleotide in the genome - only the particular one assayed, at a fraction of the cost of sequencing.

\footnotetext{
Address for correspondence. Elizabeth K. Speliotes, MD, PhD, MPH, Divisions of Gastroenterology, and Computational Medicine and Bioinformatics, Department of Internal Medicine, University of Michigan, 1150 W. Medical Center Dr., Room 6520, Ann Arbor, Michigan 48109-5682, espeliot@med.umich.edu.
} 
Approximately 2.8 million SNP variants seen more than once in individuals have been cataloged for their frequency in 290 individuals of European, Asian, and African ancestries by the human HapMap Project. ${ }^{7}$ Data from the HapMap Project revealed that the SNPs are not completely randomly distributed between individuals (Fig. 1). Individuals have stretches of similarity (correlation or linkage disequilibrium) between adjacent SNPs, so that the genotype at any one of these correlated SNPs is sufficient to predict the genotype at the others. ${ }^{8}$ Thus not every SNP needs to be genotyped to capture genetic variation in some regions of the genome, which makes it economically possible with limited genotyping to test correlated SNPs in the genome (often called haplotypes) for effects (associations) with human traits. However, if an association is found with a haplotype, because of the linkage disequilibrium, it is often not possible to determine which one of the correlated SNPs is causal.

\section{Association Studies}

Once genotyped, SNPs can be tested for association with any phenotype (or trait) of interest. Initially, scientists tested SNPs near genes that they thought were likely involved in affecting a phenotype. These were called candidate gene association studies. Although some of the SNPs associated with traits did replicate on further analyses, the vast majority did not. These studies were also limited to studying variation near genes already thought to be associated with the trait of interest and thus their novelty was limited. The development of affordable commercial genotyping products allowed simultaneous genotyping of many thousands of these SNPs across the genome of an individual. These genotyped SNPs could then be tested without bias for association with phenotypes of interest and the genome-wide association study (GWAS) era began. Thus far, much of the candidate gene and GWAS work has been performed in individuals of European ancestry to take advantage of the fact that they on average have longer areas of linkage disequilibrium than individuals of Asian or African ancestry. Therefore, even with limited genotyping, one can determine whether a SNP exists in high correlation to any genotyped SNP that affected those traits in individuals of European ancestry due to this large degree of linkage disequilibrium.

Over the last few years, we have seen an explosion of GWASs with human traits. As of December 2013, more than 11,000 SNP variants had been identified that associate with human traits with $p$ values of association of less than $10^{-5}$ from 1,751 curated publications assaying more than 100,000 SNPs $^{9}$ (Fig. 2) across 17 empirically determined and ontologybased classifications of human traits. This is extremely exciting, for it begins to reveal our own susceptibility to developing certain traits and diseases, which hopefully will give us a better understanding of our own biology and improve our ability to alter disease outcomes in the future. The strength of GWASs is that they can narrow down the regions of the genome responsible for human traits more finely than we have ever been able to identify previously, but much work still needs to be done to take what we are learning from GWASs and use it to change clinical medical practice. 


\section{Interpreting GWASs: False-Positives in Association Studies}

Over the past few years, much has been learned about how both candidate gene and GWASs need to be performed to minimize false-positive results. ${ }^{10} \mathrm{~A}$ false-positive association, also known as a type I error, can occur because of poor genotyping quality, stratification, or inadequate consideration of multiple hypothesis testing. As part of proper quality control, samples are often removed if they do not genotype well, are contaminated, there is a sample mix-up, or there is an unexpected duplicate. Single nucleotide polymorphisms that perform poorly, either by having a low genotype call rate of less than $95 \%$, that do not have good homozygous and heterozygous clustering of genotypes, or that do not pass Hardy-Weinberg equilibrium in controls are also excluded from analysis. Some studies now take the hundreds of thousands of SNPs in the genome and use them to impute (predict) the genotype of nearby SNPs (using HapMap linkage disequilibrium information), so that data can be combined across different genotyping platforms. When studies use imputed data, the quality of the SNP imputation also needs to be taken into account with low-quality imputed SNPs being excluded from analysis. Even with all of these quality checks in place, spurious associations can still occur; some propose replication of all findings in at least one other independent sample. ${ }^{11}$ Further, individuals who do not come from the same population as most of the rest of the sample need to either be removed from analyses or their ancestry variation corrected with covariates that take the population structure into account. ${ }^{12}$ Finally, given that many hundreds of thousands of SNPs are tested for association with a particular phenotype, statistical consideration for this multiple hypothesis testing needs to be taken into account. ${ }^{13}$ For standard genotyping arrays (where $\sim 1$ million independent tests are done), a $p$ value of $5 \times 10^{-8}$ is now the standard threshold to pass for a variant to be considered associated with a trait. ${ }^{14}$ However, there are custom genome-wide products (exome chips, immunochips, metabochips) where the number of comparisons may be higher or lower than the standard products, and this must be statistically taken into account when evaluating the $p$ values of association.

\section{Interpreting GWASs: False-Negatives in Association Studies}

Much has been learned about how a true signal might not be detected in a candidate gene and GWASs. ${ }^{10}$ False-negative associations (type II errors) can occur because of lack of power, gaps in the genomic coverage of the arrays, or because they require interactions with other variants or specific environmental factors that do not exist or are not uniformly present in the samples being analyzed. Power can be increased by increasing the number of samples used to detect an association. ${ }^{15}$ Often this is done by screening by GWAS in a discovery population, then taking the top associating variants, genotyping them in replication cohorts, and combining the data via meta-analysis. ${ }^{16}$ Power can be decreased by the presence of phenotype heterogeneity. Phenotype heterogeneity occurs when a trait is made up of multiple subtraits that we cannot separate from the main trait; therefore, the cases are not all homogeneous. In this scenario, a SNP that is associated with one subtrait, but not the others, will not have as strong of an association signal as it would have had it been separated from the other cases. ${ }^{17}$ However, even if such heterogeneity is present, with enough samples, if a variant does reach genome-wide significance it can still be detected. Further, many variants that are truly associated with the phenotype may not reach genome-wide significance and for 
these, we need further evidence to substantiate their association. This evidence is usually in the form of replication studies to increase the number of samples and power to detect a signal. In addition, after variants with real associations are discovered (e.g., for ulcerative colitis) they can be tested for effects in related phenotypes (primary sclerosing cholangitis) with less of a statistical burden than in genome-wide scans. Another reason false-negatives may exist is that the current genotyping arrays do not cover the entire genome perfectly, often missing low-frequency SNPs and SNPs that are not in strong linkage disequilibrium with other SNPs, particularly in non-European ancestries where genetic diversity is even higher than in Europeans. ${ }^{18,19}$ Such gaps in the coverage of these arrays will be lessening with newer genotyping products that allow assessment of even more SNPs and with nextgeneration sequencing, where all the nucleotides will now be assayed, becoming more affordable. Finally, genetic variants may not be picked up at genome-wide significance levels and in a GWAS because they require interaction with another genetic variant or with some environmental factor that is not consistently present in the samples assayed to give it enough of a signal to be detected. Methods are currently being developed to try to detect such signals. ${ }^{20,21}$

\section{From Association to Cause}

Even when a genetic variant is found that best associates (lead SNP) with a trait at genomewide significance, that variant may not necessarily be the causal variant at that locus. This is because that variant may simply be in linkage disequilibrium with the causal variant that just by random statistical chance was not found to be the best associated SNP, or alternatively was not assayed well because it is rare or in low linkage disequilibrium with the genotyped SNP. Finding the causal variant is important because it can then be used for functional studies in vitro and for genetic testing across ancestries where linkage disequilibrium may differ. Furthermore, many lead SNPs do not fall within the coding regions of genes, but instead fall in noncoding regions of the genome. ${ }^{22}$ This then requires an additional step of trying to identify which gene(s) those variants affect. This is of course important if we are to identify genes that underlie many disease traits so that we can target them for therapeutic intervention. Reassuringly, two-thirds of the time the best-associated variant is likely to affect the nearest gene to that variant. ${ }^{23}$ This suggests that when we do find associations of SNPs with traits, examining the genes near the SNPs will very likely lead us in the right direction for making new discoveries related to the biology of the trait of interest.

\section{Hype versus Hope}

As the reader will see in the articles that follow, GWASs related to liver disease have undoubtedly increased our understanding of the pathophysiology of many conditions. This information is already starting to be used to better classify people into risk categories and may be used for better diagnosis and management of liver diseases in the future. In the next few years, with genotyping of even larger populations of individuals, more genetic variants will be discovered that associate with liver disease. Furthermore, with next generation sequencing, we will also discover rarer variants that the GWAS studies currently do not assess well, that have effects intermediate between the strong effects of Mendelian conditions, and the relatively weak effects of the many common variants we are identifying 
by GWASs. Indeed, some of the associations found by GWASs, when further characterized by direct sequencing, may lead us to rarer causal variants of even stronger effect that are responsible for the association. These causal variants can then be used to better assess one's personal risk of developing disease and will allow us to move from the relatively crude epidemiologically based medicine that we practice today to the more personalized medicine of the future. Part of this future will involve prospective trials that help us to better understand what risk factors people with particular genotypes should avoid to remain disease free (gene-environment interaction studies) and what treatments work best for their particular cause of the disease (tailored treatments). The path from initial association to finding the causal genetic variant at these loci, to proving which genes these variants affect, to targeting them for therapeutic intervention and tailoring medical advice and treatment will obviously take time (Fig. 3) but is both the hype and the hope of the current GWAS era.

\section{Acknowledgments}

This work was supported by NIH K23DK080145, the Doris Duke Medical Foundation, The University of Michigan Biological Scholars Program, The Central Society for Clinical Research, and the Internal Medicine Department at the University of Michigan.

\section{References}

1. Cargill M, Altshuler D, Ireland J, et al. Characterization of single-nucleotide polymorphisms in coding regions of human genes. Nat Genet. 1999; 22(3):231-238. [PubMed: 10391209]

2. Halushka MK, Fan JB, Bentley K, et al. Patterns of single-nucleotide polymorphisms in candidate genes for blood-pressure homeostasis. Nat Genet. 1999; 22(3):239-247. [PubMed: 10391210]

3. Li WH, Sadler LA. Low nucleotide diversity in man. Genetics. 1991; 129(2):513-523. [PubMed: 1743489]

4. Wang DG, Fan JB, Siao CJ, et al. Large-scale identification, mapping, and genotyping of singlenucleotide polymorphisms in the human genome. Science. 1998; 280(5366):1077-1082. [PubMed: 9582121]

5. Kruglyak L, Nickerson DA. Variation is the spice of life. Nat Genet. 2001; 27(3):234-236. [PubMed: 11242096]

6. Reich DE, Gabriel SB, Altshuler D. Quality and completeness of SNP databases. Nat Genet. 2003; 33(4):457-458. [PubMed: 12652301]

7. Gibbs RA, Belmont JW, Hardenbol P, et al. International HapMap Consortium. The International HapMap Project. Nature. 2003; 426(6968):789-796. [PubMed: 14685227]

8. International HapMap Consortium. A haplotype map of the human genome. Nature. 2005; 437(7063):1299-1320. [PubMed: 16255080]

9. Welter D, MacArthur J, Morales J, et al. The NHGRI GWAS Catalog, a curated resource of SNPtrait associations. Nucleic Acids Res. 2014; 42:D1001-D1006. (Database issue). [PubMed: 24316577]

10. McCarthy MI, Abecasis GR, Cardon LR, et al. Genome-wide association studies for complex traits: consensus, uncertainty and challenges. Nat Rev Genet. 2008; 9(5):356-369. [PubMed: 18398418]

11. Chanock SJ, Manolio T, Boehnke M, et al. NCI-NHGRI Working Group on Replication in Association Studies. Replicating genotype-phenotype associations. Nature. 2007; 447(7145):655660. [PubMed: 17554299]

12. Price AL, Patterson NJ, Plenge RM, Weinblatt ME, Shadick NA, Reich D. Principal components analysis corrects for stratification in genome-wide association studies. Nat Genet. 2006; 38(8): 904-909. [PubMed: 16862161]

13. Rice TK, Schork NJ, Rao DC. Methods for handling multiple testing. Adv Genet. 2008; 60:293308. [PubMed: 18358325] 
14. Pe'er I, Yelensky R, Altshuler D, Daly MJ. Estimation of the multiple testing burden for genomewide association studies of nearly all common variants. Genet Epidemiol. 2008; 32(4): 381-385. [PubMed: 18348202]

15. Park J-H, Wacholder S, Gail MH, et al. Estimation of effect size distribution from genome-wide association studies and implications for future discoveries. Nat Genet. 2010; 42(7):570-575. [PubMed: 20562874]

16. Speliotes EK, Willer CJ, Berndt SI, et al. MAGIC; Procardis Consortium. Association analyses of 249,796 individuals reveal 18 new loci associated with body mass index. Nat Genet. 2010; 42(11): 937-948. [PubMed: 20935630]

17. Darabi H, Humphreys K. Single- and multi-locus association tests incorporating phenotype heterogeneity. Hum Hered. 2011; 71(1):11-22. [PubMed: 21325863]

18. Anderson CA, Pettersson FH, Barrett JC, et al. Evaluating the effects of imputation on the power, coverage, and cost efficiency of genome-wide SNP platforms. Am J Hum Genet. 2008; 83(1):112119. [PubMed: 18589396]

19. Pe'er I, de Bakker PI, Maller J, Yelensky R, Altshuler D, Daly MJ. Evaluating and improving power in whole-genome association studies using fixed marker sets. Nat Genet. 2006; 38(6):663667. [PubMed: 16715096]

20. Manning AK, LaValley M, Liu CT, et al. Meta-analysis of gene-environment interaction: joint estimation of SNP and SNP $\times$ environment regression coefficients. Genet Epidemiol. 2011; 35(1): 11-18. [PubMed: 21181894]

21. Zhang X, Huang S, Zou F, Wang W. Tools for efficient epistasis detection in genome-wide association study. Source Code Biol Med. 2011; 6(1):1. [PubMed: 21205316]

22. Willer CJ, Speliotes EK, Loos RJ, et al. Wellcome Trust Case Control Consortium. Genetic Investigation of ANthropometric Traits Consortium. Six new loci associated with body mass index highlight a neuronal influence on body weight regulation. Nat Genet. 2009; 41(1):25-34. [PubMed: 19079261]

23. Lango Allen H, Estrada K, Lettre G, et al. Hundreds of variants clustered in genomic loci and biological pathways affect human height. Nature. 2010; 467(7317):832-838. [PubMed: 20881960] 


\section{A. European Ancestry}

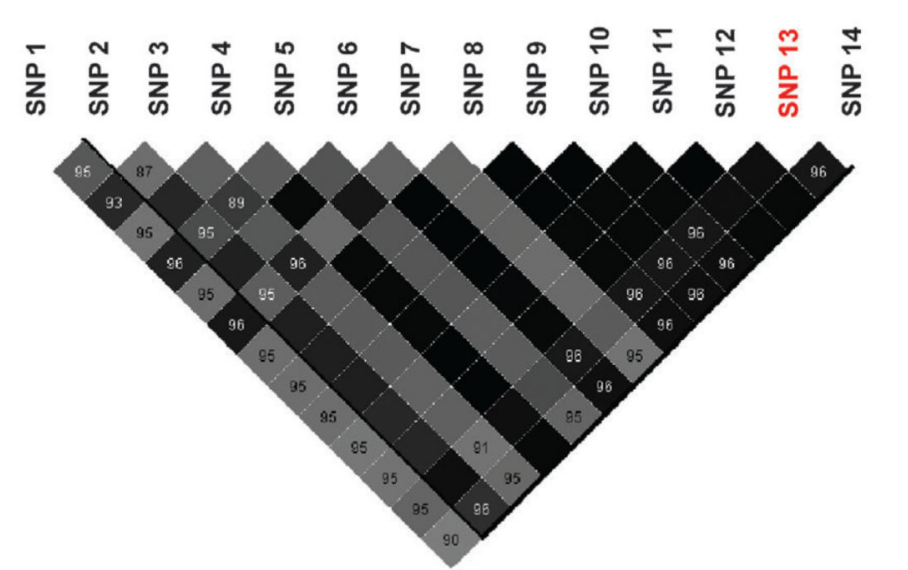

\section{B. African Ancestry}

Fig. 1.

How linkage disequilibrium (LD) affects identifying an association signal and narrowing down the casual variant. Plots of the $\mathrm{R}^{2}$ (squared correlation of the genotypes) between adjacent single nucleotide polymorphisms (SNPs) in the same region of individuals of European (CEU) and African (YRI) ancestries from the HapMap. The color of the squares under the SNPs represents the $\mathrm{R}^{2}$ between the two SNPs with black $=1$ and white $=0$. If the SNP in the region that causes a disease is SNP 13 (red), genotyping practically any of the nearby SNPs in individuals of European ancestry would lead to us discovering its association with disease because the genotype at any of these is highly correlated with the genotype at SNP 13. This is not the case in individuals of African ancestry, where we would have to genotype SNP 13 itself or at best SNP 14, 9, or 3, which have the closest correlations to the genotype at SNP 13 but are still not that strongly correlated with it. Although identifying the association signal in individuals of European ancestry with limited genotyping is more likely given the high degree of LD, a disadvantage is that because of the high degree of LD, it cannot tell us which one of the SNPs in the region is really causal. For identifying the causal variant, further studies in individuals of other ancestries like Africans, where the LD is lower, is required or functional studies if a functional assay is available. It is also possible, however, that the real signal could be coming from a rare variant that has not even been genotyped in the CEU or YRI populations, and this will only be uncovered by more directed sequencing studies of these regions. 

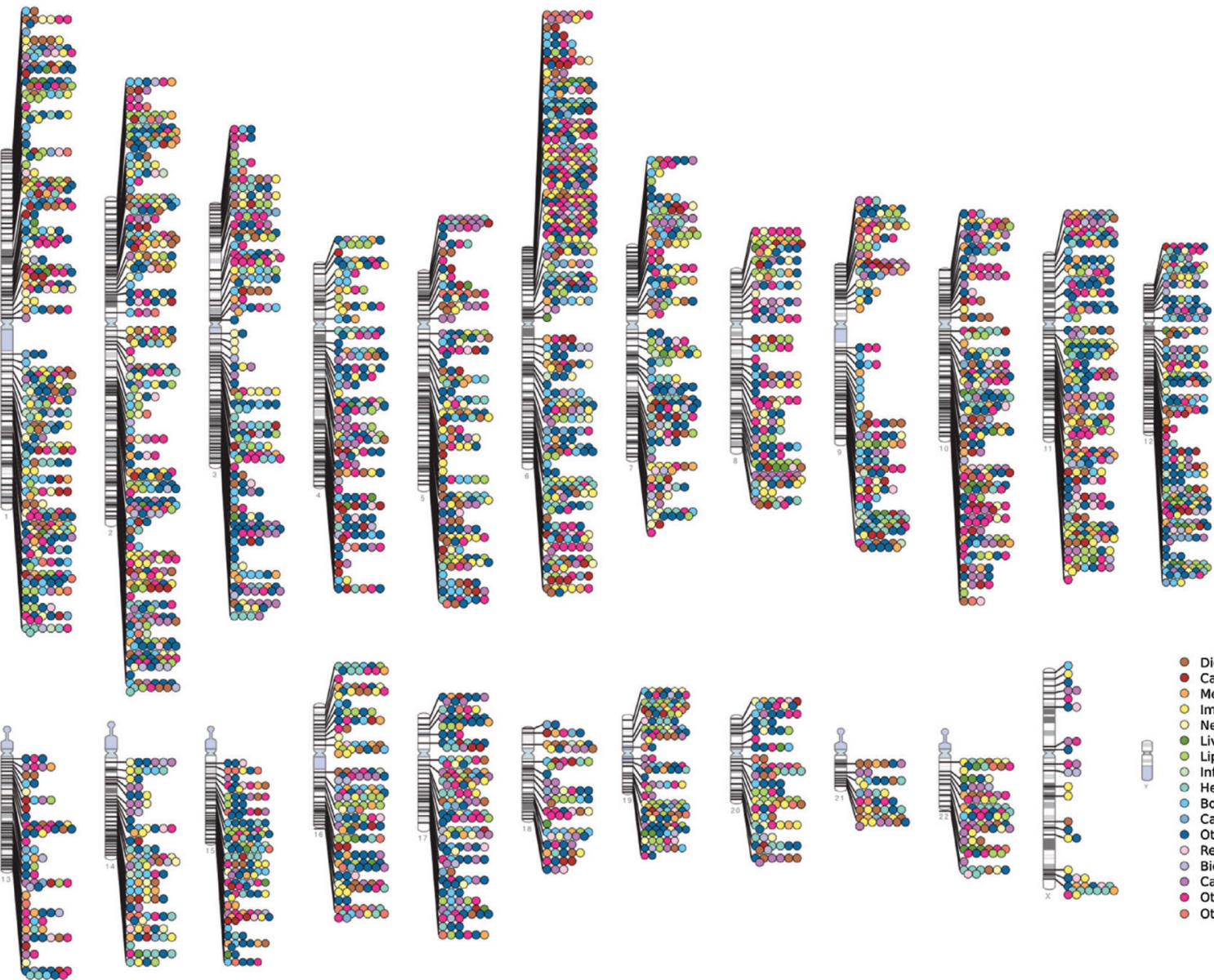

- Digestive system disease - Cardiovascular disease

Immune systeme

- Nervous system disease

- Liver enzymem measurement

- Lipid or lipoprotein measurement

- Inflammatory marker measurement

- Body measurement

- Cardiovascular measurment

- Other measurement

- Biological process

- Biological process

- Other disease

Fig. 2.

Published genome-wide associations through December 2013. The position along the 22 autosomes and the $\mathrm{X}$ and $\mathrm{Y}$ chromosomes are shown for genetic associations that have reached genome-wide significance with p values $<5 \times 10^{-8}$. The phenotype category that the genetic variant associates with is demarcated by the color of the circle. The chromosomes are shown with their cytological "bands" in gray. Details on the associations can be obtained at www.genome.gov/GWAStudies. 
A. Association region to causal variant

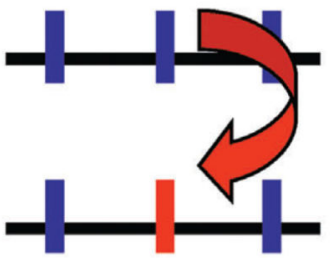

C. Gene to function

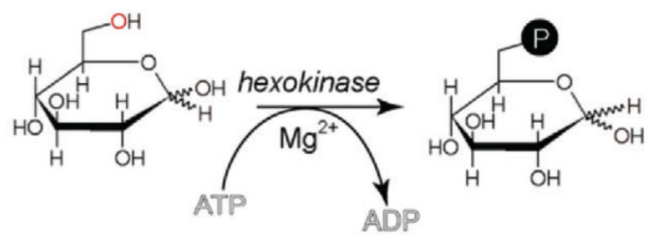

B. Variant to gene

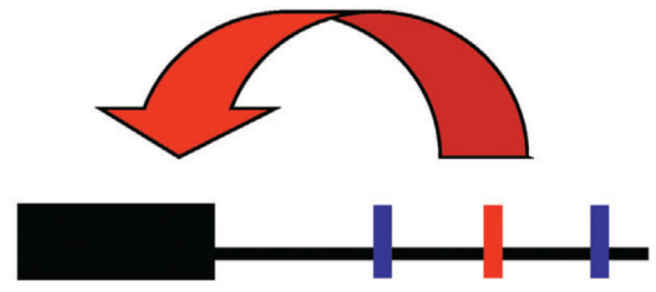

D. Function to pathway

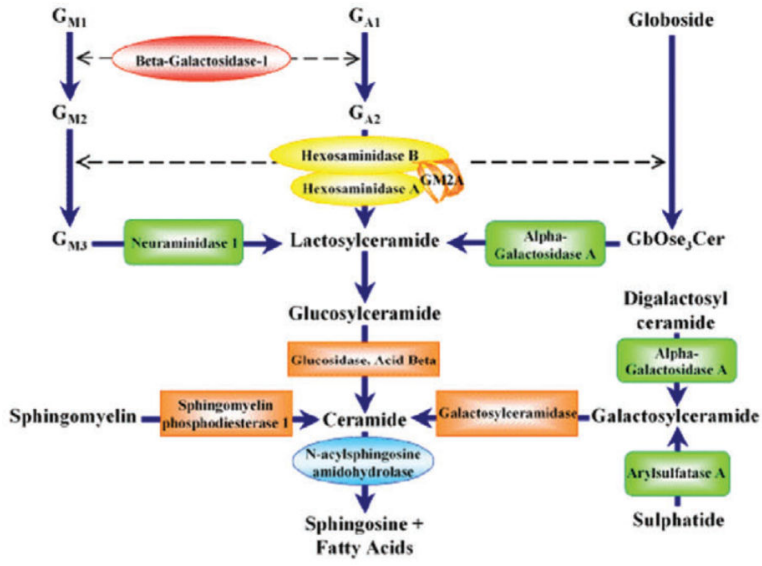

Fig. 3.

From association to cause. Multiple steps are currently required to get us from the associations we are finding by GWASs today to the actual causes of the diseases they relate to. (A) First we must go from the region of association to identifying the actual causal variant. (B) We must then go from the causal variant to identifying the gene or genes those causal variants affect. (C) We must then determine the function of the genes these variants affect, and (D) understand how those functions relate to pathways that affect the disease of interest (and possibly other diseases) to be able to make the most informed decisions about how to combat the disease specifically without causing undesirable side effects. 
Table 1

Genetic terms often used in genome-wide association study analyses

\begin{tabular}{|c|c|}
\hline Term & Definition \\
\hline Genetic & Relating to DNA \\
\hline Phenotype & A measured trait or disease \\
\hline Genetic code & The series of nucleotides $(\mathrm{A}, \mathrm{C}, \mathrm{G}, \mathrm{T})$ that make up DNA \\
\hline Genetic variant & Nucleotide or structural differences in parts of the genome that differ between individuals \\
\hline Nucleotide & The adenine, cytosine, guanine, and thymine components that compose DNA \\
\hline $\begin{array}{l}\text { Single nucleotide } \\
\text { polymorphism (SNP) }\end{array}$ & Variation in nucleotide sequence at a particular spot in the genetic code between individuals \\
\hline Allele & An alternative form of a nucleotide at a spot in the genome \\
\hline Variance & The diversity in the measurement of a particular trait \\
\hline Heritability & The proportion of the variation in a trait that is genetically influenced \\
\hline Genotyping & Determining the nucleotide present in one particular point in the genome \\
\hline Sequencing & Determining all of the nucleotides present in regions of the genome \\
\hline HapMap & Characterized variation in millions of genetic variants across the genome in diverse ancestries \\
\hline Linkage disequilibrium (LD) & The nonrandom association of particular alleles in the genome \\
\hline Haplotype & A group of nearby alleles that are inherited together \\
\hline $\mathrm{CEU}$ & Individuals of Northern European ancestry (from Utah specifically) in HapMap \\
\hline YRI & Individuals of African ancestry from Yoruba (Nigeria specifically) in HapMap \\
\hline GWAS & $\begin{array}{l}\text { Genome-wide association study; where many thousands of variants across the genome } \\
\text { are tested for association with a trait of interest }\end{array}$ \\
\hline Association & The presence of a particular genotype with a phenotype above and beyond statistical chance \\
\hline Stratification & $\begin{array}{l}\text { The presence of individuals of different ancestries in a sample which when not accounted } \\
\text { for can lead to spurious genetic associations }\end{array}$ \\
\hline Multiple hypothesis testing & $\begin{array}{l}\text { When more than one hypothesis is being tested for statistical association } \\
\text { (i.e., more than } 1 \text { SNP) }\end{array}$ \\
\hline Clustering & $\begin{array}{l}\text { Individuals with particular genotypes will give off more similar detection patterns and thus } \\
\text { cluster together as they are being detected }\end{array}$ \\
\hline Hardy-Weinberg equilibrium & $\begin{array}{l}\text { A measure of the frequencies of each genotype should be in a population that has reached } \\
\text { equilibrium-if SNPs do not genotype properly they often are out of equilibrium and are thus } \\
\text { eliminated from analyses }\end{array}$ \\
\hline Impute & $\begin{array}{l}\text { To use known SNP genotypes and LD patterns from reference populations to predict the } \\
\text { genotype at nearby SNPs }\end{array}$ \\
\hline Power & $\begin{array}{l}\text { The chance of detecting an effect of a certain size in a sample of particular } \\
\text { size-range } 0 \text { to } 100 \%\end{array}$ \\
\hline Arrays & Products that contain probes to assess multiple SNPs simultaneously \\
\hline Lead SNP & The SNP initially found to best associate with a trait of interest statistically \\
\hline Causal SNP & The SNP that actually causes variation in the trait of interest \\
\hline Prevalence & Total number of cases of a disease at a particular time \\
\hline Incidence & $\begin{array}{l}\text { Total number of new cases of a disease per a certain number of people surveyed } \\
\text { over a particular period }\end{array}$ \\
\hline Nonsynonymous variant & A variant that changes the amino acid structure of a protein \\
\hline Synonymous variant & A variant that does not change the amino acid structure of a protein \\
\hline
\end{tabular}

Semin Liver Dis. Author manuscript; available in PMC 2017 April 11. 


\begin{tabular}{|l|l|}
\hline Term & Definition \\
\hline Missense variant & A variant that changes the amino acid structure of a protein \\
\hline Gain-of-function mutation & $\begin{array}{l}\text { When a variant leads to a protein having a new function that the normal } \\
\text { protein does not have }\end{array}$ \\
\hline eQTL & $\begin{array}{l}\text { Expression QTL studies where genetic variants are tested for whether they are } \\
\text { associated with changes in gene expression }\end{array}$ \\
\hline Functional variant & A variant that leads to a noticeable phenotype \\
\hline Locus & A region of the genome \\
\hline Gene & A part of the genome that make a protein of particular function \\
\hline Odds ratio & $\begin{array}{l}\text { A measure of effect size, describing the strength of association or no nindependence } \\
\text { between two binary data values }\end{array}$ \\
\hline Attributable risk & $\begin{array}{l}\text { Difference in the rate of a condition between an exposed population and an } \\
\text { unexposed population }\end{array}$ \\
\hline Positive predictive value & Proportion of individuals with positive test results who truly have the disease/response \\
\hline Negative predictive value & Proportion of individuals with negative test results who truly do not have the disease/response \\
\hline
\end{tabular}

